\title{
A Case of Choroidal Neovascularization Secondary to Unilateral Retinal Pigment Epithelium Dysgenesis
}

\author{
Tsuyoshi Shimoyama ${ }^{a, b} \quad$ Hisanori Imai $^{a, b} \quad$ Shigeru Honda ${ }^{a} \quad$ Akira Negi $^{a}$ \\ ${ }^{a}$ Division of Ophthalmology, Department of Organ Therapeutics, Kobe University \\ Graduate School of Medicine, and 'bepartment of Ophthalmology, Kobe Kaisei Hospital, \\ Kobe, Japan
}

\section{Key Words}

Unilateral retinal pigment epithelium dysgenesis · Anti-VEGF · Bevacizumab · Choroidal neovascularization - Triamcinolone · Fundus autofluorescence

\begin{abstract}
Aim: To report a case of choroidal neovascularization secondary to unilateral retinal pigment epithelium dysgenesis (URPED), which was resistant to posterior subtenon injection of triamcinolone acetonide (STTA) and intravitreal bevacizumab injection (IVB). Case Report: An 8-year-old boy was referred to us because of a unilateral unique clinical appearance on funduscopic examination in his left eye (OS). A geometric lesion at the retinal pigment epithelium level of the interpapillomacular area was disclosed OS. The optic nerve was slightly hyperemic OS. Findings from the right fundus examination were normal. Based on these characteristic findings, he was diagnosed as having URPED. Best corrected Landolt ring chart visual acuity (BCVA) was 1.0 in both eyes. Twenty-three months after the first visit, the patient presented with visual disturbance OS. Funduscopic examination showed an expansion of the geometric lesion and the development of a subfoveal choroidal neovascularization (CNV). BCVA was 0.4 OS. Two-time STTA $(40 \mathrm{mg} / 1 \mathrm{ml})$ was performed the onset of CNV and 6 months later, and additional IVB $(1.25 \mathrm{mg} / 0.05 \mathrm{ml})$ was done 10 months later for the treatment of CNV, but the geometric lesion and CNV were resistant to the treatment and continued to expand. Seven years after the first visit, the geometric lesion and the CNV kept expanding steadily. Conclusion: URPED is a rare clinical entity, and the prognosis of this disease is still unclear. The visual prognosis may depend on whether CNV fully develops.
\end{abstract}


Shimoyama et al.: A Case of Choroidal Neovascularization Secondary to Unilateral Retinal Pigment Epithelium Dysgenesis

\section{Introduction}

Unilateral retinal pigment epithelium dysgenesis (URPED) is a rare clinical entity [1-3]. It is characterized by a unilateral occurrence in young patients and consists of a round affected area with a distinct scalloped margin of reticular retinal pigment epithelium (RPE) hyperplasia, mid-lesion lacunae of RPE hyperplasia, central thinning and atrophy of RPE. The findings of fundus autofluorescence (FAF) and fluorescein angiography (FA) are the most important when diagnosing URPED. The visual prognosis of URPED is still unclear, but it may depend on whether choroidal neovascularization (CNV) will develop [1].

In this report, we present a case of CNV associated with URPED, which was resistant to the treatment with posterior subtenon injection of triamcinolone acetonide (STTA) and intravitreal bevacizumab injection (IVB).

\section{Case Report}

An 8-year-old boy was referred to us because of a unilateral unique funduscopic appearance in the left eye (OS). Funduscopic findings revealed a geometric lesion at the RPE level of the interpapillomacular area, contiguous with the slightly hyperemic optic nerve (fig. 1). The lesion was composed of marginal RPE hyperplasia and atrophic RPE in its center. Findings from the right fundus examination were normal. Based on these characteristic findings, the patient was diagnosed with URPED. Best corrected Landolt ring chart visual acuity (BCVA) was 1.0 in both eyes. Twenty-three months after the first visit, he presented with visual disturbance OS. Funduscopic examination showed enlargement of the lesion and development of subfoveal CNV (fig. 2). FA findings revealed hypofluorescence from RPE hyperplasia, hyperfluorescence from RPE atrophy and dye leakage from the optic nerve and CNV (fig. 2). Optical coherence tomography (OCT) revealed type $2 \mathrm{CNV}$, but the exudative change was not obvious (fig. 2). The patient's BCVA was 0.4 OS. Two-time STTA (40 mg/1 $\mathrm{ml}$ ) was performed at the onset of CNV and 6 months later. Additional IVB $(1.25 \mathrm{mg} / 0.05 \mathrm{ml})$ was performed 10 months later for the treatment of CNV. Both STTA and IVB were performed with the written informed consent from both the patient and his father as well as with an approval of the institutional review board of Kobe University of Medicine and the Tenets of the Declaration of Helsinki. Despite the treatment, the geometric lesion and CNV were resistant to the treatment and expanded gradually. Seven years after the first visit, the geometric lesion and the CNV kept expanding steadily, and new CNV developed at the inferior retina (fig. 3).

\section{Discussion}

Cohen et al. [2] first reported 4 cases of this unique fundus lesion in 2002. In their study, 2 of 4 cases were complicated by CNV. Finally, in 2009, they proposed naming this disorder 'URPED' and reported that 2 of 9 cases were affected by CNV [1]. We believe that this is the first follow-up report which can provide new clinical information about this disease because Cohen et al. [1, 2] did not mention the course of CNV treatment.

In our case, CNV did not respond to both STTA and IVB. Systemic steroids, immunosuppressants or additional STTA and IVB might be effective to achieve the quieting effect of this highly active $\mathrm{CNV}$, but we did not impose them because the patient was still experiencing periods of growth. Generally, regardless of the treatment, CNV suppresses eventually with 
Shimoyama et al.: A Case of Choroidal Neovascularization Secondary to Unilateral Retinal Pigment Epithelium Dysgenesis

RPE proliferation, which is one of the remodeling processes of wound repairing. In our case, we could not observe RPE proliferation around CNV during the follow-up period. On the contrary, new CNV developed at the inferior retina. Furthermore, the geometric lesion was significantly enlarged over the RPE hyperplasia. These findings may suggest that the congenital RPE malfunction, which spreads across a wide area of the fundus, is the underlying reason for the development of this disease.

Our case is unique because we observed the hyperemic optic nerve OS at the first visit. In the previous report, Cohen et al. [1] stated that the affected area is usually contiguous with the optic nerve, but they did not mention the condition of the optic nerve itself. It is possible that the inflammation of the optic nerve is one of the triggers in the development of this unique pattern of the fundus.

To summarize, we propose that patients who develop CNV secondary to URPED should be informed carefully and always be followed up for treatment-resistant CNV.

\section{References}

$\checkmark 1$ Cohen SY, Fung AE, Tadayoni R, Massin P, Barbazetto I, Berthout A, Gayet P, Meunier I, Yannuzzi LA: Unilateral retinal pigment epithelium dysgenesis. Am J Ophthalmol 2009;148:914-919.

-2 Cohen SY, Massin P, Quentel G: Clinicopathologic reports, case reports, and small case series: unilateral, idiopathic leopard-spot lesion of the retinal pigment epithelium. Arch Ophthalmol 2002;120:512-516.

-3 Berthout A, Malthieu D, Taboureau E, Milazzo S: Unilateral idiopathic leopard-spot lesion of the retinal pigment epithelium: a fibroglial tractional complication (in French). J Fr Ophtalmol 2008;31:716.
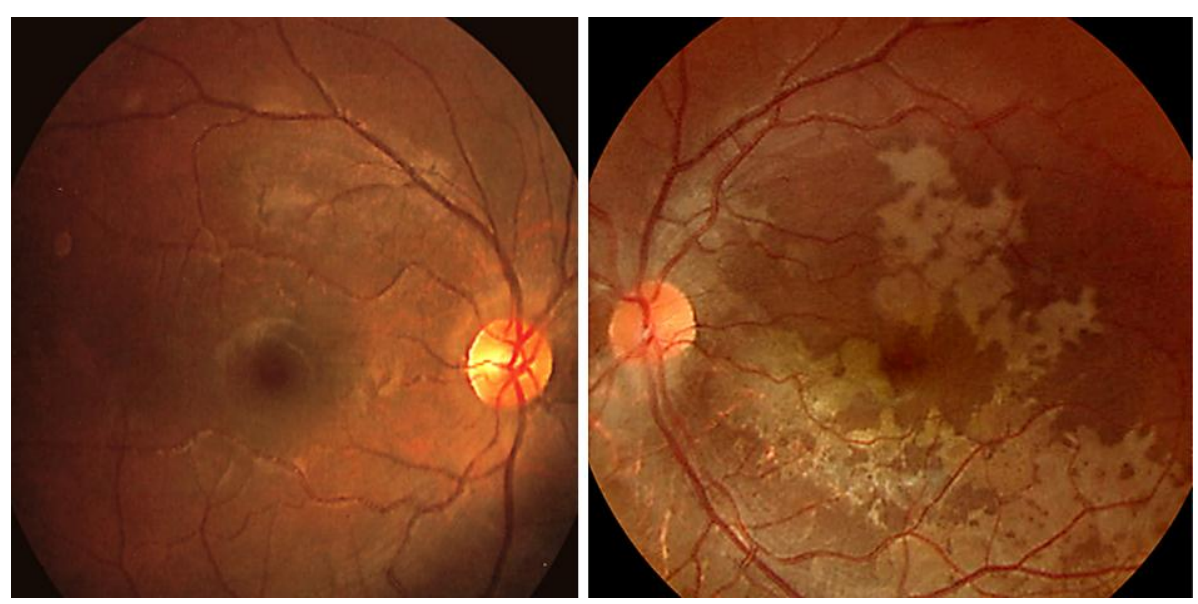

Fig. 1. Funduscopic findings at the first visit. Funduscopic findings OS revealed a geometric lesion at the RPE level of the interpapillomacular area, contiguous with the slightly hyperemic optic nerve. The lesion was composed of marginal RPE hyperplasia and atrophic RPE in its center. Findings from the right fundus examination were normal. 


\section{Case Reports in Ophthalmology}

\begin{tabular}{l|l}
\hline Case Rep Ophthalmol 2014;5:34-37 \\
\hline DOI: 10.1159/000358426 & $\begin{array}{l}@ 2014 \text { S. Karger AG, Basel } \\
\text { www.karger.com/cop }\end{array}$ \\
\hline
\end{tabular}

Shimoyama et al.: A Case of Choroidal Neovascularization Secondary to Unilateral Retinal Pigment Epithelium Dysgenesis
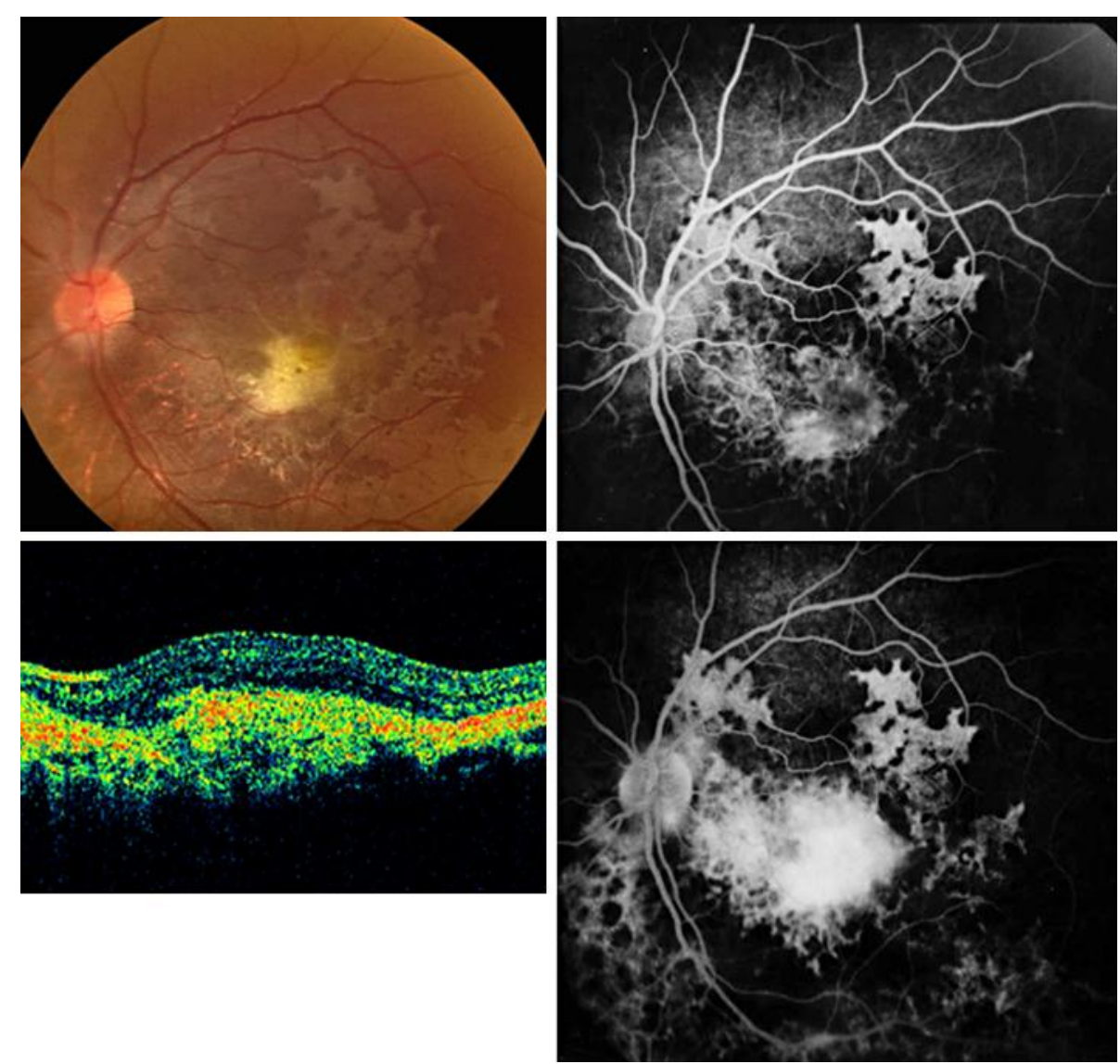

Fig. 2. Twenty-three months after the first visit, the enlargement of the lesion and the development of subfoveal CNV were observed (upper left: funduscopic findings, lower left: OCT findings). FA findings revealed dye leakage from the optic nerve and CNV (upper right: early-phase image, upper left: late-phase image).
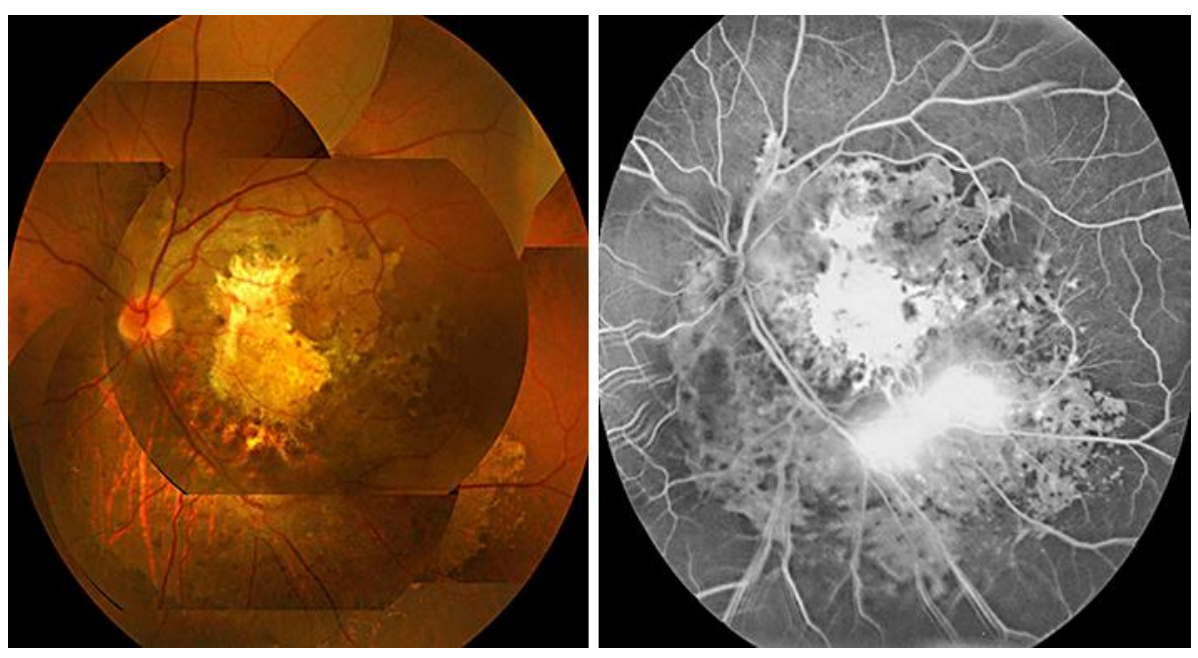

Fig. 3. Seven years after the first visit, the geometric lesion and CNV kept expanding steadily and new CNV developed at the inferior retina (upper: panoramic funduscopic findings, bottom: panoramic FA findings). 